IP $\rightleftharpoons$ B

\title{
Espécies madeireiras da Amazônia: riqueza, nomes populares e suas peculiaridades
}

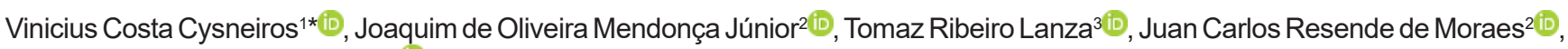 \\ Otávio José Magalhães Samor (iD
}

${ }^{1}$ Universidade Federal do Paraná, Rua Lothário Meisnner, 632, Jardim Botânico, Campus Botânico, CEP 80210-170, Curitiba, PR, Brasil

2Universidade Federal Rural do Rio de Janeiro, BR 465, Km 07, Campus Universitário, CEP 23890-000, Seropédica, RJ, Brasil

3Universidade Estadual Paulista Júlio de Mesquita Filho, Rua Doutor José Barbosa de Barros, 482, Bairro Jardim Paraíso, CEP 18610-307, Botucatu, SP, Brasil

${ }^{4}$ Dossel Florestal, Rua Coronel Castro, 237, Bairro Porto, CEP 28740-000, Conceição de Macabu, RJ, Brasil

"Autor correspondente:

vccysneiros.florestal@gmail.com

Termos para indexação:

Gestão florestal

Biodiversidade

Nomenclatura botânica

Index terms:

Forest management

Biodiversity

Botanical nomenclature

Histórico do artigo:

Recebido em 02/02/2018

Aprovado em 21/05/2018

Publicado em 13/12/2018

doi: 10.4336/2018.pfb.38e201801567
Resumo - Nos inventários florestais comerciais na Amazônia, o processo de identificação botânica pode envolver diversos erros e acarretar prejuízos ao manejo florestal. Um erro comum decorre da variação dos nomes populares, que apresentam peculiaridades regionais e podem ser atribuídos a diversas espécies simultaneamente. Este estudo tem como objetivos analisar a riqueza de espécies madeireiras exploradas na Amazônia brasileira e verificar as variações regionais dos nomes populares das principais espécies, visando identificar padrões e casos de fidelidade. Para isso, foram consultadas informações de espécies comerciais atualmente exploradas e passíveis de exploração em 10 planos de manejo florestal sustentável em diferentes categorias e regiões da Amazônia brasileira. As análises evidenciaram elevada riqueza de espécies comerciais, com baixa similaridade entre as espécies, indicando particularidades locais quanto às espécies de interesse. Os nomes populares apresentaram ampla variação, com duas tendências distintas: utilização de um mesmo nome popular para diversas espécies e utilização de diversos nomes populares para uma única espécie. Foram detectados casos de fidelidade para os nomes populares das principais espécies madeireiras. No entanto, os nomes populares apresentam forte relação com gêneros e famílias botânicas, podendo variar intensamente quando a análise requer identificação em nível de espécie.

\section{Amazonian timber species: richness, popular names and their peculiarities}

\begin{abstract}
The process of botanical identification in commercial forest inventories in Amazon may involve several errors and entail losses to forest management. A common error is the variation of popular names, which have regional peculiarities and can be attributed to several species simultaneously. This study aims to analyze the richness of timber species exploited in the Brazilian Amazon and verify the regional variations of popular names of main species, aiming to identify patterns and cases of fidelity. Information on exploited and potential commercial trees was explored in 10 sustainable forest management plans in different categories and regions of the Brazilian Amazon. The analyzes showed high richness of commercial species, with low similarity among species, indicating local peculiarities for main species. Popular names presented a wide variation, with two distinct tendencies: use of the same popular name for several botanical species and use of several popular names for a single botanical species. Cases of fidelity were detected for popular names of main timber species. However, popular names are strongly related to botanical genera and families, and may vary intensely when the analysis requires identification at species level.
\end{abstract}




\section{Introdução}

Mesmo representando o bioma com maior diversidade biológica no planeta, a Floresta Amazônica vem sofrendo impactos negativos, devido principalmente ao acelerado e desordenado processo de ocupação humana (Obermuller et al., 2011). Assim, o governo brasileiro busca conciliar o desenvolvimento da região com a conservação das florestas, tendo o manejo florestal sustentável como uma das melhores opções (Veríssimo et al., 2002). No entanto, a conservação e o manejo das florestas tropicais requerem a identificação precisa das espécies-alvo, o que demanda alto nível de conhecimento taxonômico e, às vezes, a presença de material fértil, dificultando essa atividade em áreas de elevada diversidade (Lang et al., 2017). A escassez de herbários e coleções botânicas de referência no vasto território da Amazônia legal ressaltam a dificuldade mencionada, tornando a identificação botânica ainda mais complexa.

Nos inventários para manejo florestal na Amazônia, o processo de identificação botânica ocorre em duas etapas: 1) em campo, onde uma equipe identifica, mensura e localiza em um sistema de coordenadas geográficas todas as árvores inclusas no critério de amostragem pré-estabelecido e os nomes das espécies são atribuídos por um parataxonomista; e 2) no escritório, onde as denominações são convertidas em nomes científicos (Baraloto et al., 2007). Esse processo pode envolver diversos erros e acarretar prejuízos ao manejo florestal, sendo que um erro comumente observado decorre da variação dos nomes populares, que apresentam peculiaridades regionais e podem ser atribuídos a diversas espécies simultaneamente (Ferreira \& Hopkins, 2004; Baraloto et al., 2007).

Este estudo teve como objetivos analisar a riqueza de espécies madeireiras comercializadas na Amazônia brasileira e verificar as variações regionais dos nomes populares das principais espécies, visando identificar padrões e casos de fidelidade. Para isso, as seguintes hipóteses foram elaboradas: 1) a exploração de uma espécie considerada comercial pode ser afetada por diversos fatores locais, conferindo particularidades florísticas e riqueza de espécies de interesse diferenciada entre planos de manejo florestal (PMF) na Amazônia; 2) os nomes populares das espécies madeireiras, mesmo variando entre regiões, apresentam fidelidade para algumas espécies de mercado consolidado e apresentam forte relação com os gêneros e famílias botânicas; 3) os principais táxons com problemas de identificação nos PMFs da Amazônia estão relacionados a gêneros e famílias botânicas de elevada riqueza e complexidade taxonômica.

\section{Material e métodos}

Para compilação das informações sobre as espécies madeireiras, foram utilizados dados de 10 planos de manejo florestal sustentável (PMFSs) localizados em diferentes regiões da Amazônia brasileira (Figura 1) e sob categorias distintas de manejo (Tabela 1), com o objetivo de captar as peculiaridades das espécies em diversos cenários do manejo florestal no Brasil. Em todos os planos, a identificação botânica foi realizada com auxílio de profissionais especializados e os materiais botânicos referentes às espécies comerciais foram tombados em herbários de instituições de pesquisa. Os planos selecionados para este estudo estão aprovados para produção madeireira, são públicos e estão disponibilizados online. Assim, foram consultadas informações de árvores exploradas e potenciais à exploração, catalogadas como comerciais, tendo registrado os nomes científico e popular e o número de indivíduos explorados e selecionados como potenciais. Após a compilação, todos os nomes científicos foram revisados de acordo com o APG IV (The Angiosperm Phylogeny Group, 2016), a fim de atualizar a nomenclatura botânica e evitar possíveis sinonímias.

A riqueza de espécies comerciais presentes nos planos de manejo foi avaliada por meio da curva de rarefação, utilizando o método de Mao Tau no programa Estimates (Colwell et al., 2011), com dados binários de presença e ausência. Para analisar a similaridade entre os planos de manejo em termos de espécies comerciais, foi empregada a análise de agrupamento com os dados binários, por meio de classificadores hierárquicos com o método Ward no pacote estatístico R. O agrupamento formado foi analisado com o objetivo de verificar a influência dos polos madeireiros sobre a preferência regional de espécies comerciais.

As espécies com identificação incompleta, identificadas apenas ao nível de gênero ou de família, foram listadas separadamente, a fim de analisar os principais grupos botânicos que geram dificuldades taxonômicas para a atividade madeireira. Esses grupos 
são pontos frágeis para o inventário de florestas de produção na Amazônia, cuja identificação incorreta pode acarretar prejuízos ecológicos e econômicos para o manejo florestal, como instabilidade de mercado e colheitas insustentáveis (Baraloto et al., 2007).

Para análise dos nomes populares, as espécies madeireiras foram separadas em dois grupos, com base em tendências distintas relatadas na literatura (Baraloto et al., 2007): 1) utilização de um mesmo nome popular para diversas espécies botânicas; e 2) utilização de diversos nomes populares para uma única espécie botânica. Assim, foram discutidas as variações e peculiaridades regionais dos nomes populares das principais espécies madeireiras na Amazônia, dando ênfase à fidelidade e aos padrões encontrados para esses nomes.

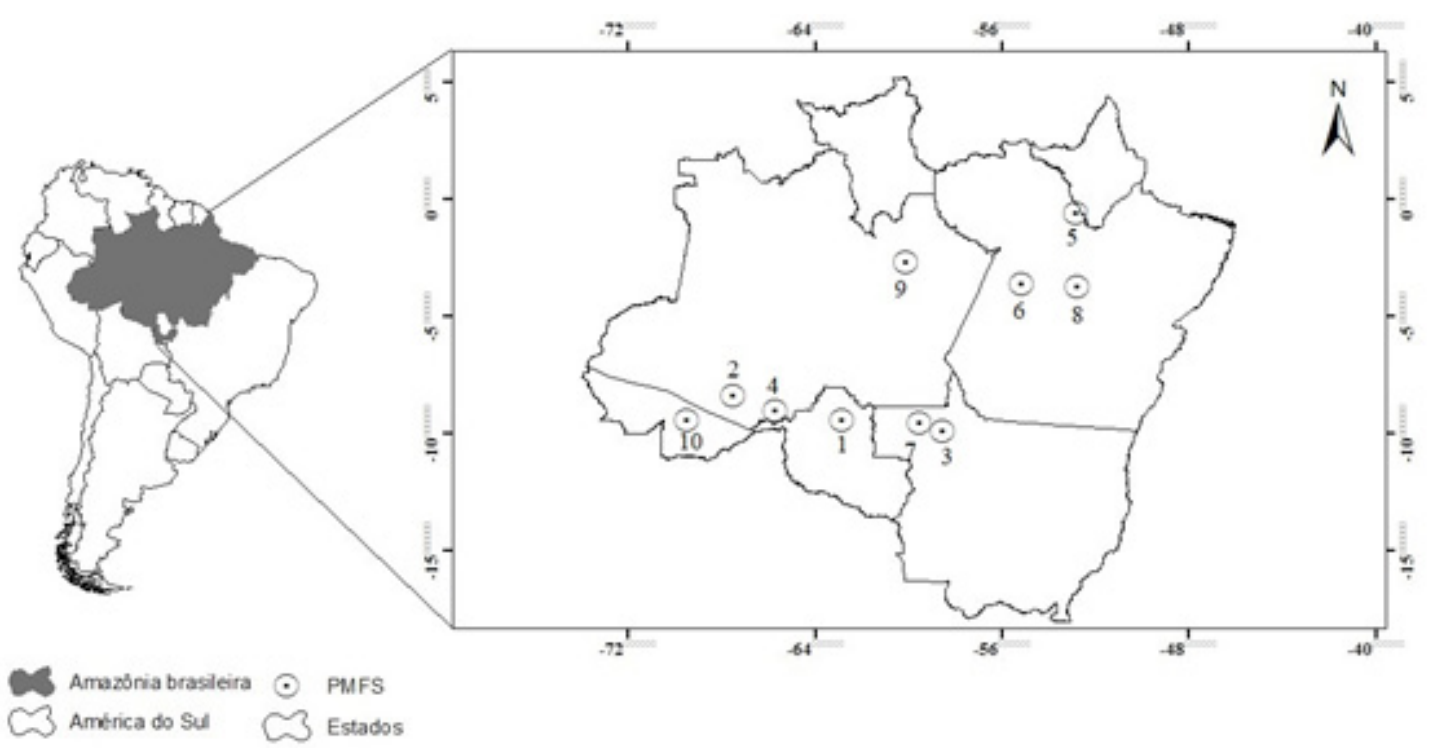

Figura 1. Localização geográfica dos planos de manejo florestal sustentável analisados na Amazônia brasileira.

Figure 1. Geographic location of the sustainable forest management plans analyzed in the Brazilian Amazon.

Tabela 1. Descrição dos planos de manejo florestal sustentável analisados na Amazônia brasileira.

Table 1. Description of the sustainable forest management plans analyzed in the Brazilian Amazon

\begin{tabular}{|c|c|c|c|c|c|c|}
\hline PMFS & Local & Estado & Categoria do manejo & Espécies & Condição das espécies & $\begin{array}{c}\text { Área de } \\
\text { estudo } \\
\text { (ha) }\end{array}$ \\
\hline 1 & Floresta Nacional do Jamari & RO & Empresarial em larga escala & 49 & Exploradas & 8.800 \\
\hline 2 & Floresta Nacional do Purus & $\mathrm{AM}$ & $\begin{array}{l}\text { Comunitário em pequena } \\
\text { escala }\end{array}$ & 34 & Licenciadas e potenciais & 50 \\
\hline 3 & Área privada & MT & Empresarial em larga escala & 27 & Exploradas & 2.354 \\
\hline 4 & Área privada & $\mathrm{AM}$ & Empresarial em média escala & 26 & Exploradas & 547 \\
\hline 5 & Vale do Jari & PA & Empresarial em larga escala & 25 & Exploradas & 3.786 \\
\hline 6 & Floresta Nacional de Altamira & PA & Empresarial em larga escala & 55 & Prioritárias para o manejo & 2.500 \\
\hline 7 & Município de Cotriguaçu & MT & Diagnostico Municipal & 23 & Exploradas & - \\
\hline 8 & Floresta Nacional do Tapajós & $\mathrm{PA}$ & Experimental & 85 & Potenciais a exploração & 64 \\
\hline 9 & $\begin{array}{l}\text { Estação Experimental de Silvicultura } \\
\text { Tropical }\end{array}$ & $\mathrm{AM}$ & Experimental & 85 & Potenciais a exploração & 96 \\
\hline 10 & Área privada & $\mathrm{AC}$ & Empresarial em larga escala & 47 & Exploradas & 4.677 \\
\hline
\end{tabular}

(-) sem informação. 


\section{Resultados}

\section{Riqueza de espécies}

Foram encontrados 195 táxons identificados ao nível de espécie, considerando 30 famílias botânicas, predominantes nos planos de manejo florestal sustentável (PMFSs) da Amazônia brasileira amostrados (Tabela 1). A família com maior número de espécies comerciais foi Fabaceae (68), seguida de Lauraceae (16), Lecythidaceae (12) e Sapotaceae (12). Dessas espécies, foram selecionadas como as mais importantes aquelas contempladas em mais da metade dos PFMSs analisados, sendo elas: Dipteryx odorata (90\%), Manilkara elata (90\%), Simarouba amara (80\%), Apuleia leiocarpa (70\%), Astronium lecointei (70\%), Goupia glabra (70\%), Bagassa guianensis (60\%), Caryocar glabrum
(60\%), Caryocar villosum (60\%), Clarisia racemosa (60\%), Enterolobium schomburgkii (60\%), Hymenaea courbaril (60\%), Dinizia excelsa (50\%) e Schizolobium parahyba var. amazonicum (50\%).

A curva de rarefação para as espécies apresentou forma crescente, sem estabilização (Figura 2), indicando a elevada riqueza de espécies comerciais nas áreas analisadas e necessidade de maior amostragem. Esses resultados são ressaltados quando comparado aos estimadores de riqueza Bootstrap e Jackknife, que estimaram respectivamente 241 e 301 espécies para a amostra em questão. O agrupamento dos PMFSs analisados (Figura 3) evidenciou a formação de apenas dois grupos similares quanto às espécies comerciais, enquanto as demais áreas encontram-se isoladas, sem semelhanças proeminentes entre as espécies exploradas.

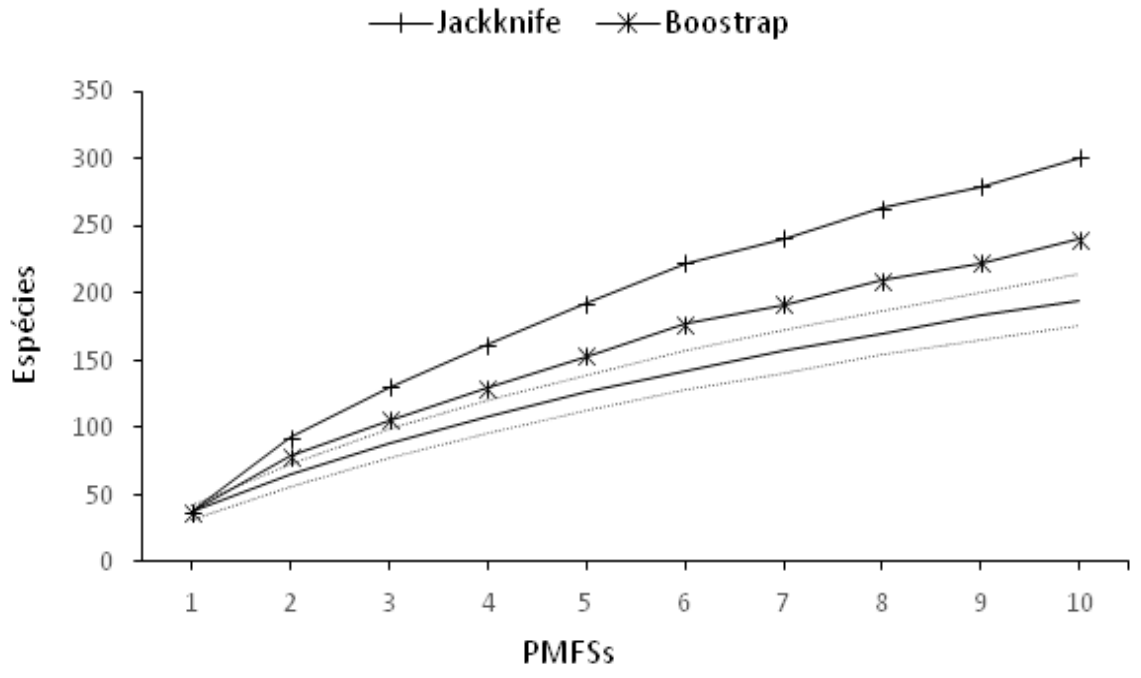

Figura 2. Curva de rarefação com seus intervalos de confiança para as espécies comerciais presentes nos planos de manejo florestal sustentável analisados na Amazônia brasileira.

Figure 2. Rarefaction curve with its confidence intervals for the commercial species present in the sustainable forest management plans analyzed in the Brazilian Amazon.

Figura 3. Agrupamento dos planos de manejo florestal sustentável analisados na Amazônia brasileira quanto à distância de similaridade de espécies comercias.

Figure 3. Grouping of sustainable forest management plans analyzed in the Brazilian Amazon as to the similarity distance of commercial species.

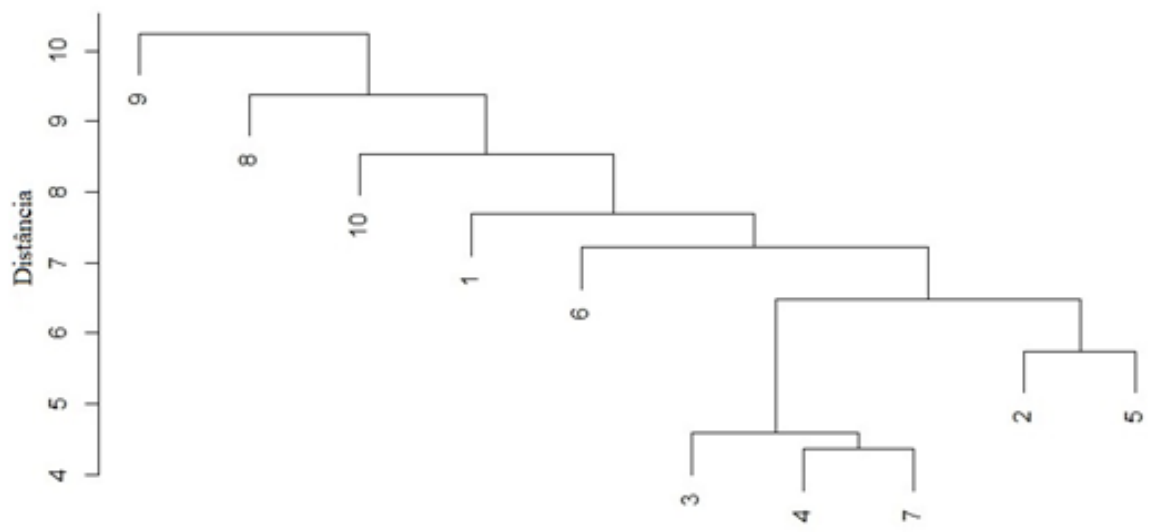


Da base de dados de espécies com identificação incompleta, foram encontrados 68 táxons, distribuídos em 34 gêneros e 15 famílias botânicas. Os gêneros mais abundantes em espécies não identificadas foram: Hymenolobium (6), Mezilaurus (5), Aspidosperma (4), Ocotea (4), Protium (4), Handroanthus (3) e Hymenaea (3).

\section{Nomes populares}

Para as 195 espécies identificadas, foram encontrados 210 nomes populares (Tabela 2), com forte variação entre as áreas. Os nomes populares que mais se repetiram, foram: angelim, louro, fava, sucupira, ucuúba e tauari, atribuídos a diversas espécies de um mesmo gênero ou família. As espécies cujos nomes populares apresentaram fidelidade foram: garapeira (Apuleia leiocarpa), muiracatiara (Astronium lecointei), pequiá (Caryocar villosum), pequiarana (Caryocar glabrum), cumaru (Dipteryx odorata), cupiúba (Goupia glabra), massaranduba (Manilkara elata) e caucho (Castilla ulei). Por outro lado, as espécies que apresentaram maior número de nomes populares foram: Enterolobium schomburgkii (5), Cariniana micrantha (4) e Brosimum rubescens (4).

Quanto aos táxons com identificação incompleta, alguns nomes populares possuem forte ligação com gêneros, como: breu (Protium.), ipê (Handroanthus), itaúba (Mezilaurus), amapá (Brosimum), tauari (Couratari), ucuúba (Virola), roxinho (Peltogyne), matámatá (Eschweilera), angelim (Hymenolobium), jatobá e jutaí (Hymenaea) e louro (Lauraceae).

Tabela 2. Lista de espécies comerciais com identificação completa nos planos de manejo florestal sustentável e seus respectivos nomes populares.

Table 2. List of commercial species with complete identification in sustainable forest management plans and their respective names.

\begin{tabular}{|c|c|c|}
\hline Espécie & Nome popular & Grupo \\
\hline Agonandra brasiliensis Miers ex Benth. \& Hook. f. & marfim-azeitona & 1 \\
\hline Alexa grandiflora Ducke & melancieira & * \\
\hline Allantoma decandra (Ducke) S. A. Mori, Y.-Y. Huang \& Prance & jequitibá & 1 \\
\hline Amburana acreana (Ducke) A. C. Sm. & cerejeira & * \\
\hline Anacardium spruceanum Benth. ex. Engl. & cajuí & - \\
\hline Andira inermis (W.Wright.) DC. & angelim-coco & 1 \\
\hline Andira micrantha Ducke & sucupira-preta & 1 \\
\hline \multirow[t]{2}{*}{ Aniba canellila (Kunth) Mez } & canelão & 1 \\
\hline & preciosa & \\
\hline Aniba hostemanniana (Ness) Mez & louro-piracuru & 1 \\
\hline Aniba parviflora (Meissn.) Mez & louro-aritu & 1 \\
\hline Aniba riparia $(\mathrm{Nees}) \mathrm{Mez}$ & louro-fofo & 1 \\
\hline Aniba rosiodora Ducke & pau-rosa & * \\
\hline Aniba williamsii O.C.Schmidt & louro-amarelo & 1 \\
\hline \multirow[t]{2}{*}{ Apuleia leiocarpa (Vogel) J. F. Macbr. } & amarelão & \\
\hline & garapeira & * \\
\hline \multirow[t]{3}{*}{ Aspidosperma desmanthum Benth. ex. Mull. Arg. } & amarelão & 2 \\
\hline & aracanga & \\
\hline & piquiá-marfim & \\
\hline Aspidosperma excelsum Benth. & carapanaúba & 1 \\
\hline Aspidosperma oblongum A. DC. & carapanaúba & 1 \\
\hline Aspidosperma spruceanum Benth. ex Mull. Arg & peroba-rosa & 1 \\
\hline Astronium graveolens Jacq. & aroeira & - \\
\hline \multirow[t]{2}{*}{ Astronium lecointei Ducke } & maracatiara & * \\
\hline & muiracatiara & \\
\hline
\end{tabular}

Continua...

Pesq. flor. bras., Colombo, v. 38, e201801567, p. 1-14, 2018 
Tabela 2. continuação.

Table 2. continuation.

\begin{tabular}{|c|c|c|}
\hline Espécie & Nome popular & Grupo \\
\hline Bagassa guianensis Aubl. & $\begin{array}{l}\text { garrote } \\
\text { tatajuba }\end{array}$ & $*$ \\
\hline Bertholletia excelsa Humb. \& Bonpl. & castanha-do-pará & * \\
\hline Bowdichia nitida Spruce ex Benth. & $\begin{array}{l}\text { sucupira-amarela } \\
\text { sucupira-pele-de-sapo }\end{array}$ & 2 \\
\hline Bowdichia virgilioides Kunth & sucupira-preta & 1 \\
\hline Brosimum acutifolium Huber & muiratinga & 1 \\
\hline Brosimum guianense (Aubl.) Huber & amapá-amargoso & 1 \\
\hline Brosimum lactescens (S. Moore) C. C. Berg. & $\begin{array}{l}\text { amapaí } \\
\text { leiteiro }\end{array}$ & 1 \\
\hline Brosimum parinarioides Ducke & amapá-doce & 1 \\
\hline Brosimum rubescens Taub. & $\begin{array}{l}\text { amapá-bravo } \\
\text { inharé } \\
\text { muirapiranga } \\
\text { pau-rainha }\end{array}$ & 1,2 \\
\hline Buchenavia grandis Ducke & tanimbuca & 1 \\
\hline Buchenavia guianensis (Aubl.) Alwan \& Stace & tanimbuca-cinzeiro & - \\
\hline Buchenavia parvifolia Ducke & tanimbuca & 1 \\
\hline Calophyllum brasiliense Cambess. & jacareúba & * \\
\hline Calycophyllum spruceanum (Benth.) K. Schum. & mulateiro & $*$ \\
\hline Carapa guianensis Aubl. & andiroba & * \\
\hline Cariniana micrantha Ducke & $\begin{array}{l}\text { castanha-de-macaco } \\
\text { corrimboque-castanheira } \\
\text { jequitibá } \\
\text { tauari-vermelho }\end{array}$ & 2 \\
\hline Caryocar glabrum (Aubl.) Pers. & pequiarana & * \\
\hline Caryocar microcarpum Ducke & pequiá & 1 \\
\hline Caryocar villosum (Aubl.) Pers. & $\begin{array}{l}\text { pequi } \\
\text { pequiá }\end{array}$ & * \\
\hline Castilla ullei Warb. & caucho & * \\
\hline Cedrela fissilis Vell. & cedro-rosa & * \\
\hline Cedrela odorata $\mathrm{L}$. & $\begin{array}{l}\text { cedro } \\
\text { cedro-rosa }\end{array}$ & 1 \\
\hline Cedrelinga cateniformis (Ducke) Ducke & $\begin{array}{l}\text { cedromara } \\
\text { cedrorana } \\
\text { cupiuba }\end{array}$ & 2 \\
\hline Ceiba pentandra (L.) Gaertn. & sumaúma & * \\
\hline Centrolobium paraense Tul. & potumuju & - \\
\hline Chrysophyllum amazonicum T. D. Penn. & abiurana-sapucaia & 1 \\
\hline Clarisia ilicifolia (Spreng.) Lanj. \& Roosberg & janitá & - \\
\hline Clarisia racemosa Ruíz \& Pav. & guariúba & * \\
\hline
\end{tabular}


Tabela 2. continuação.

Table 2. continuation.

\begin{tabular}{|c|c|c|}
\hline Espécie & Nome popular & Grupo \\
\hline & oiticica & \\
\hline Copaifera langsdorffii Desf. & copaíba & $1, *$ \\
\hline Copaifera multijuga Hayne & copaíba & $1, *$ \\
\hline Copaifera reticulata Ducke & copaíba & $1, *$ \\
\hline Cordia goeldiana Huber & freijó & 1 \\
\hline Couma utilis (Mart.) Mull. Arg. & sorveira & 1 \\
\hline \multirow[t]{2}{*}{ Couratari guianensis Aubl. } & tauari & 1 \\
\hline & tauari-peludo & \\
\hline Couratari macrosperma A. C. Sm. & tauari & 1 \\
\hline Couratari oblongifolia Ducke \& Kunth & tauari & 1 \\
\hline \multirow[t]{2}{*}{ Couratari stellata A. C. Sm. } & embireira & 1 \\
\hline & tauari & \\
\hline Couratari tauari O. Berg. & tauari & 1 \\
\hline \multirow[t]{2}{*}{ Dialium guianense (Aubl.) Sandwith } & jutaí-mirim & 2 \\
\hline & tamarindo & \\
\hline Dimorphandra coccinea Ducke & faveira & 1 \\
\hline \multirow[t]{3}{*}{ Dinizia excelsa Ducke } & angelim-vermelho & 2 \\
\hline & angelim-pedra & \\
\hline & faveira-ferro & \\
\hline \multirow[t]{3}{*}{ Diplotropis purpurea (Rich.) Amshoff } & sucupira & 2 \\
\hline & sucupira-amarela & \\
\hline & sucupira-preta & \\
\hline Diplotropis rodriguesii H.C. Lima & sucupira-preta & 1 \\
\hline Diplotropsis triloba Gleason & sucupira-chorona & - \\
\hline Dipteryx alata Vogel & cumarurana & 1 \\
\hline Dipteryx magnifica Ducke & cumarurana & 1 \\
\hline \multirow[t]{2}{*}{ Dipteryx odorata (Aubl.) Willd. } & cumaru & * \\
\hline & cumaru-ferro & \\
\hline Dipteryx punctata (Blake) Amshoff & cumaru & 1 \\
\hline Endopleura uchi (Huber) Cuatrec. & uchi & * \\
\hline Enterolobium maximum Ducke & fava-timbauva & - \\
\hline \multirow[t]{5}{*}{ Enterolobium schomburgkii (Benth.) Benth. } & fava-de-rosca & 2 \\
\hline & fava-orelha-de-macaco & \\
\hline & faveira-dura & \\
\hline & orelha-de-macaco & \\
\hline & orelha-de-vovó & \\
\hline Eperua bijuga Mart. Ex Benth. & cocão & - \\
\hline Eperua schomburgkiana Benth. & apazeiro & - \\
\hline Eriotheca globosa (Aubl.) A.Robyns & paineira-rosa & - \\
\hline Erisma bicolor Ducke & cinzeiro & - \\
\hline Erisma fuscum Ducke & cedrilho & 1 \\
\hline
\end{tabular}


Tabela 2. continuação.

Table 2. continuation.

\begin{tabular}{|c|c|c|}
\hline Espécie & Nome popular & Grupo \\
\hline \multirow[t]{2}{*}{ Erisma uncinatum Warm. } & cedrinho & 1 \\
\hline & quarubarana & \\
\hline \multirow[t]{2}{*}{ Eschweilera coriacea (DC.) S. A. Mori } & matá-matá & 1 \\
\hline & matá-matá-amarelo & \\
\hline Eschweilera grandiflora (Aubl.) Sandwith & matá-matá & 1 \\
\hline Euxylophora paraensis Huber & amarelão & 1 \\
\hline Ficus castellviana Dugand & figueira & - \\
\hline Glycydendron amazonicum Ducke & mirindiba-doce & 1 \\
\hline \multirow[t]{2}{*}{ Goupia glabra Aubl. } & azedinha & - \\
\hline & cupiúba & $*$ \\
\hline \multirow[t]{2}{*}{ Guarea guidonia (L.) Sleumer } & jitó & 1 \\
\hline & marinheiro & \\
\hline \multirow[t]{2}{*}{ Handroanthus impetiginosus (Mart.ex DC.) Mattos } & ipê & 2 \\
\hline & ipê-roxo & \\
\hline \multirow[t]{3}{*}{ Handroanthus incanus (A. H. Gentry) S. O. Grose } & ipê & 2 \\
\hline & ipê-amarelo & \\
\hline & pau-d`arco & \\
\hline \multirow[t]{2}{*}{ Handroanthus serratifolius (Vahl) Nichols } & ipê & 2 \\
\hline & ipê-amarelo & \\
\hline Hevea brasiliensis (Willd. ex A. Juss.) Mull. Arg. & seringueira & * \\
\hline Huberodendron swietenioides (Gleason) Ducke & embira-bero & - \\
\hline Hura crepitans $\mathrm{L}$. & assacu & $*$ \\
\hline \multirow[t]{2}{*}{ Hymenaea courbaril $\mathrm{L}$. } & jatobá & 1,2 \\
\hline & jutaí-açu & \\
\hline Hymenaea intermedia Ducke & jatobazinho & 1,2 \\
\hline $\begin{array}{l}\text { Hymenaea oblongifolia var. palustris (Ducke) Y. T. Lee \& } \\
\text { Langenh. }\end{array}$ & jatobá & 1,2 \\
\hline Hymenaea parvifolia Huber & jutaí-mirim & 1,2 \\
\hline Hymenaea reticulata Ducke & jatobá & 1,2 \\
\hline Hymenolobium excelsum Ducke & angelim-da-mata & 1 \\
\hline \multirow[t]{2}{*}{ Hymenolobium heterocarpum Ducke } & angelim-da-mata & 1,2 \\
\hline & angelim-pedra & \\
\hline Hymenolobium modestum Ducke & angelim & 1 \\
\hline \multirow[t]{2}{*}{ Hymenolobium petraeum Ducke } & angelim & 1 \\
\hline & angelim-pedra & \\
\hline Iryanthera coriacea Ducke & ucuúba & 1 \\
\hline \multirow[t]{2}{*}{ Iryanthera juruensis Warb. } & apunã & 1 \\
\hline & uccúba-punã & \\
\hline \multirow[t]{2}{*}{ Iryanthera macrophylla (Benth.) Warb. } & ucuúba & 1 \\
\hline & ucuúba-vermelha & \\
\hline Iryanthera paradoxa (Schwacke) Warb. & arurá-vermelho & 1 \\
\hline
\end{tabular}

Continua... 
Tabela 2. continuação.

Table 2. continuation.

\begin{tabular}{|c|c|c|}
\hline Espécie & Nome popular & Grupo \\
\hline \multirow[t]{2}{*}{ Jacaranda copaia (Aubl.) D. Don } & caroba & 2 \\
\hline & caroba & \\
\hline \multirow[t]{3}{*}{ Laetia procera (Poepp.) Eichler } & mandioqueira & 2 \\
\hline & pau-jacaré & \\
\hline & periquiteira-amarela & \\
\hline Lecythis lurida (Miers) S. A. Mori & jarana & 1 \\
\hline Lecythis pisonis Cambess & castanha-sapucaia & 1 \\
\hline Licaria chrysophylla (Meisn.) Kosterm. & louro-aritu & 1 \\
\hline \multirow[t]{3}{*}{ Licaria crassifolia (Poir.) P. L. R. Moraes } & canela-caxeta & 2 \\
\hline & louro-bosta & \\
\hline & louro-canela & \\
\hline Luehea cymulosa Spruce ex Benth. & açoita-cavalo & - \\
\hline Maclura tinctoria $($ L.) D. Don ex Steud & amoreira & - \\
\hline Macrolobium campestre Huber & arapari & - \\
\hline \multirow[t]{2}{*}{ Manilkara bidentata (A. DC.) A. Chev. } & maçaranduba & * \\
\hline & massaranduba & * \\
\hline \multirow[t]{2}{*}{ Manilkara elata (Allemão ex Miq.) Monach. } & maçaranduba & * \\
\hline & massaranduba & \\
\hline Manilkara inundata (Ducke) Ducke & parajú & 1 \\
\hline Martiodendron elatum (Ducke) Gleason & tamarindo & - \\
\hline Mezilaurus itauba (Meissn) Taub. ex. Mex & itaúba & $*$ \\
\hline \multirow[t]{3}{*}{ Mezilaurus lindaviana Schwacke \& Mez } & itaúba & 2 \\
\hline & itaúba-amarela & \\
\hline & itaubão & \\
\hline Mezilaurus synandra (Mez) Kosterm. & itaúba & 1 \\
\hline Micropholis melinoniana Pierre & currupixá & 1 \\
\hline \multirow[t]{2}{*}{ Micropholis venulosa (Mart. \& Eichler) Pierre. } & abiu-mangabinha & 1 \\
\hline & currupixá & \\
\hline \multirow[t]{2}{*}{ Minquartia guianensis Aubl. } & acariquara & 1 \\
\hline & acariquara-roxa & \\
\hline Mouriri brevipes Hook. & goiabão & 1 \\
\hline Myroxylon balsamum (L.) Harms & cabreúva & - \\
\hline Myroxylon peruiferum $\mathrm{L}$. f. & cabreúva-parda & - \\
\hline Ocotea cymbarum Kunth & louro-amarelo & 1 \\
\hline Ocotea guianensis Aubl. & louro-seda & 1 \\
\hline \multirow[t]{2}{*}{ Ocotea longifolia Kunth } & canela & 1 \\
\hline & louro-preto & \\
\hline Ocotea nigrescens Vicentini & louro-preto & 1 \\
\hline Ormosia paraensis Ducke & tento & 1 \\
\hline Osteophloeum platyspermum (Spruce ex A. DC.) Warb. & ucuúba-d'agua & $*$ \\
\hline
\end{tabular}

Continua... 
Tabela 2. continuação.

Table 2. continuation.

\begin{tabular}{|c|c|c|}
\hline Espécie & Nome popular & Grupo \\
\hline Parahancornia fasciculata (Poir.) Benoist & amapá-roxo & 1 \\
\hline Parapiptadenia rigida (Benth.) Brenan & timborana & - \\
\hline \multirow[t]{2}{*}{ Parkia multijuga Benth. } & bajão & 2 \\
\hline & fava-de-tucupi & \\
\hline Parkia paraensis Ducke & bandarra & - \\
\hline \multirow[t]{3}{*}{ Parkia pendula (Willd.) Benth. ex Walp. } & faveira & 2 \\
\hline & fava-bolota & \\
\hline & visgueiro & \\
\hline Peltogyne catingae Ducke & roxinho & 1 \\
\hline Peltogyne excelsa Ducke & violeta & 1 \\
\hline Peltogyne paniculata Benth. & roxinho & 1 \\
\hline Peltogyne paradoxa Ducke & coataquicaua & - \\
\hline Peltogyne venosa (Vahl) Benth. & roxão & 1 \\
\hline Piptadenia gonoacantha (Mart.) J. F. Macbr & timborana & 1 \\
\hline Platymiscium filipes Benth. & macacaúba & 1 \\
\hline Platymiscium pinnatum var. ulei (Harms) Klitg. & macacaúba & 1 \\
\hline Platymiscium trinitatis Benth. & macacaúba & 1 \\
\hline Pouteria bilocularis (H. K. A.Wink1.) Baehni & goiabão & 1 \\
\hline Pouteria caimito (Ruiz \& Pav.) Radlk. & abiu e abiurana & 1 \\
\hline \multirow[t]{2}{*}{ Pouteria elegans (A. DC.) Baehni } & guarajá & * \\
\hline & guarajá-pedra & \\
\hline Pouteria eugeniifolia (Pierre) Baehni & abiurana & 1 \\
\hline \multirow[t]{2}{*}{ Pouteria guianensis Aubl. } & abiu & 1 \\
\hline & abiu-vermelho & \\
\hline Pouteria torta subsp. tuberculata (Sleumer) T. D. Penn. & abiu & 1 \\
\hline Protium altsonii Sandwith & breu-branco & 1 \\
\hline Protium apiculatum Swart & breu-gigante & 1 \\
\hline Protium hebetatum Daly & breu-vermelho & 1 \\
\hline Protium heptaphyllum (Aubl.) Marchand & amescla & 1 \\
\hline Protium nitidifolium (Cuatrec.) Daly & breu-pitomba & 1 \\
\hline Protium robustum (Swart) D. M. Porter & breu & 1 \\
\hline Pseudopiptadenia suaveolens (Miq.) J. W. Grimes & fava-timborana & 1 \\
\hline Pterocarpus officinalis Jacq. & jutaí-cica & 1 \\
\hline \multirow[t]{3}{*}{ Qualea paraensis Ducke } & cambará-rosa & 1,2 \\
\hline & madioqueira & \\
\hline & mandioqueira-aspera & \\
\hline Roupala montana Aubl. & louro-faia & 1 \\
\hline \multirow[t]{2}{*}{ Ruizterania albiflora (Warm.) Marcano-Berti } & mandioqueira-áspera & 1 \\
\hline & mandioqueira-lisa & \\
\hline Ruizterania cassiquiarensis (Spruce ex Warm.) Marcano-Berti & mandioqueira-lisa & 1 \\
\hline \multirow[t]{2}{*}{ Schefflera morototoni (Aubl.) Maguire } & mandiocão & 2 \\
\hline & morototó & \\
\hline
\end{tabular}


Tabela 2. continuação.

Table 2. continuation.

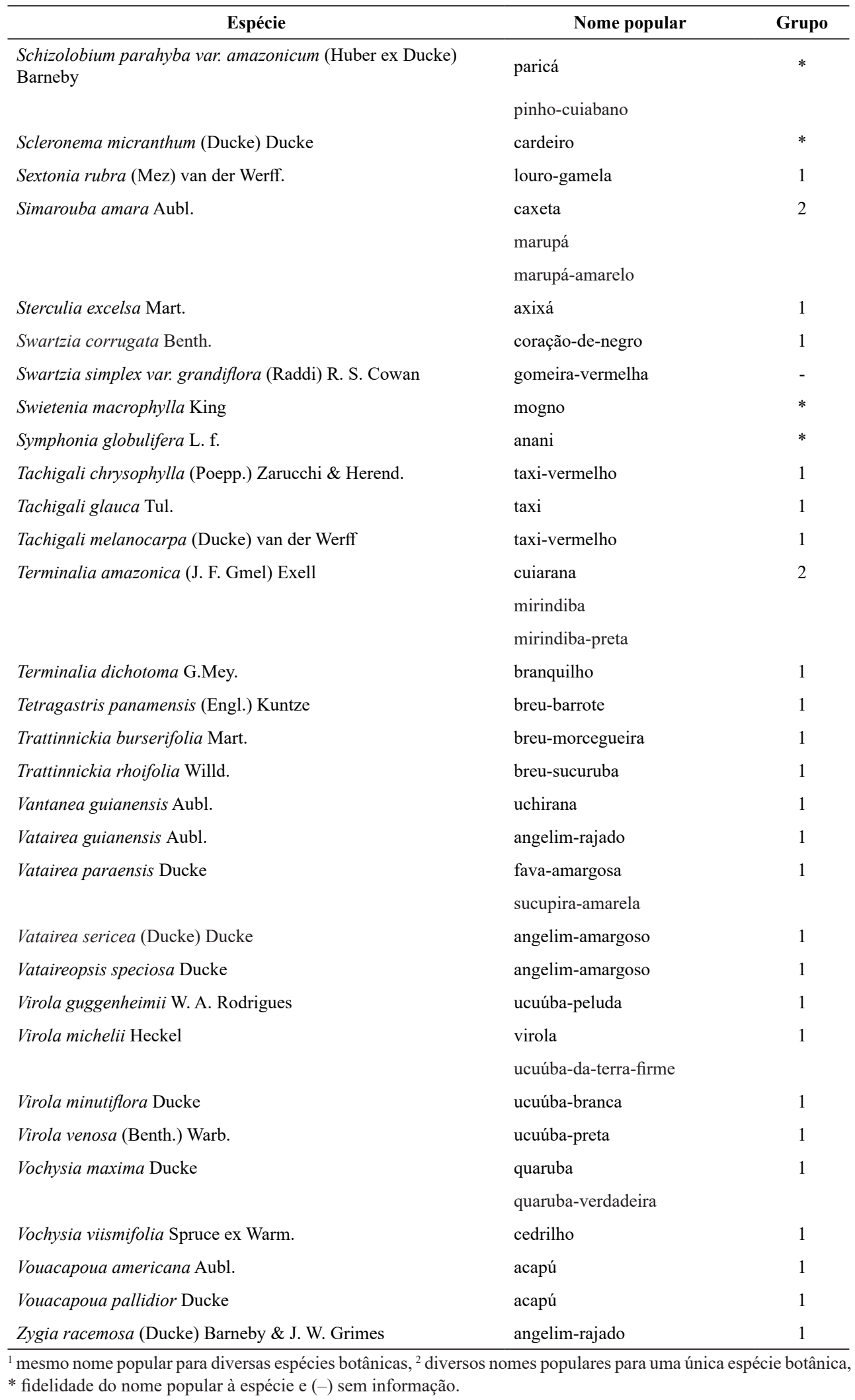




\section{Discussão}

\section{Riqueza de espécies}

A floresta amazônica é conhecida por apresentar a maior diversidade de espécies arbóreas dos trópicos, com forte influência dos fatores ambientais sobre os padrões regionais de diversidade. No entanto, em escalas locais, diversos fatores podem atuar como limitantes dessa diversidade (Ter Steege et al., 2003; Toledo et al., 2011). Esses complexos padrões de diversidade indicam tendência crescente da riqueza de espécies com o acréscimo de novas áreas de manejo e elevada riqueza de espécies estimada pelos estimadores Bootstrap e Jackknife (Figura 2).

Esses padrões de diversidade também podem afetar a dissimilaridade entre as áreas, onde é rara a formação de grupos similares quanto às espécies comerciais (Figura 3). Assim, a destacada variação dos padrões de diversidade para diferentes escalas (locais e regionais) reflete as diferenças regionais entre as espécies comerciais, como a demanda do mercado local e a estrutura populacional das espécies nas florestas alvo do manejo.

As 14 principais espécies comerciais listadas neste trabalho apresentam ampla distribuição geográfica na Amazônia brasileira (Ferreira \& Hopkins, 2004; Obermuller et al., 2011). Essa característica, associada à qualidade de suas madeiras e a presença abundante dessas espécies nas florestas da Amazônia, favorecem seu manejo por meio de PMFSs e, por isso, estão dentre as principais espécies madeireiras amazônicas.

Os gêneros que se destacaram em táxons com identificação incompleta são citados dentre os de maior riqueza de espécies arbóreas na Amazônia brasileira e suas madeiras são altamente apreciadas pelo mercado madeireiro (Ribeiro et al., 1999; Ferreira \& Hopkins, 2004; Baraloto et al., 2007; Obermuller et al., 2011). Assim, esses gêneros são considerados pontos chave para o manejo florestal e, por possuírem mercados consolidados, sua identificação correta se torna crucial para evitar prejuízos, tanto econômicos como ambientais.

\section{Nomes populares}

Evidentemente, existem padrões regionais para os nomes populares que, sobretudo, estão relacionados aos dialetos locais (Baraloto et al., 2007). Os nomes populares com intensa repetição, que caracterizam o grupo 1 , fazem alusão às espécies de interesse comercial contempladas com frequência nos PMFSs e, em muitos casos, estão relacionados aos gêneros ou famílias com complexidade taxonômica e elevada riqueza de espécies. As espécies cujos nomes populares apresentaram fidelidade são amplamente conhecidas e exploradas por toda Amazônia, com mercado nacional e internacional consolidado (Ferreira \& Hopkins, 2004; Obermuller et al., 2011), não aparentando problemas quanto à identificação. Do mesmo modo, são caracterizadas as espécies com diversos nomes populares, presentes no grupo 2. No entanto, as espécies desse grupo também apresentam ampla distribuição geográfica, ocorrendo com frequência em todo território analisado.

Os táxons com identificação incompleta estão relacionados às espécies, gêneros e famílias de interesse comercial e de complexidade taxonômica (Ribeiro et al., 1999; Ferreira \& Hopkins, 2004). Vale ressaltar que o nome popular angelim, que apresentou fidelidade ao gênero Hymenolobium na análise de táxons incompletos, pode estar atribuído aos diversos gêneros da família Fabaceae (Ferreira \& Hopkins, 2004), como constatado para as espécies com identificação completa. Apesar da intensa variação regional, os resultados obtidos indicam que os nomes populares apresentam forte relação com gêneros e famílias botânicas, podendo variar intensamente quando a análise requer identificação ao nível de espécie.

A presença de um nome com forte relação a um gênero de interesse comercial, associado a um sufixo, é uma peculiaridade dos nomes populares das espécies madeireiras na Amazônia. Esse sufixo geralmente faz alusão a alguma característica da espécie, como: cor da madeira (sucupira-preta), cor da casca externa (ucuúba-vermelha), flores (ipê-amarelo) e densidade e durabilidade da madeira (angelim-pedra e faveira-ferro). Outro sufixo comum é rana, de origem indígena e que significa falso, faz alusão às espécies com características semelhantes às espécies de alto valor comercial e amplamente conhecidas nos mercados madeireiros, como itaubarana, quarubarana e cedrorana.

Um componente ainda negligenciado nos planos de manejo das florestas tropicais é a identificação botânica das espécies alvo (Baraloto et al., 2007). A atenção imediata para resolução desse problema é necessária, do contrário haverá consequências para gestão das florestas tropicais (Janzen, 2004; Baraloto 
et al., 2007). Atualmente, técnicas inovadoras baseadas em espectroscopia das folhas estão sendo empregadas para identificação precisa de espécies arbóreas na Amazônia (Lang et al., 2017), apresentando inúmeras aplicabilidades para as pesquisas cientificas. Assim, o direcionamento dessas pesquisas à identificação das principais espécies e gêneros de interesse comercial, aliado à contínua capacitação dos técnicos responsáveis pela atividade, trará avanço para o setor florestal, aprimorando uma questão chave para a prática do manejo sustentável nas florestas tropicais.

Perante a elevada riqueza de espécies comerciais e o predomínio de grupos com elevada complexidade taxonômica nas florestas da Amazônia, é ressaltada a necessidade de profissionais especializados envolvidos nas atividades de identificação botânica e a constante qualificação dos parataxonomistas de campo. Esses merecem especial atenção, pois são um elemento chave para busca e disponibilização de informações sobre a biodiversidade, em prol do desenvolvimento sustentável (Janzen, 2004). Uma opção para os agentes do manejo florestal são as parcerias com universidades e instituições de pesquisa, que podem ser proveitosas para todas as partes envolvidas, como demostrado por Baraloto et al. (2007).

As análises apresentadas têm caráter exploratório e objetivo de embasar a discussão sobre a riqueza de espécies madeireiras e as peculiaridades de seus nomes populares na Amazônia. Devido à extensão territorial e às particularidades da flora Amazônica, é necessário a expansão da base de dados para a realização de uma análise mais completa e abrangente. Para isso, é sugerida a disponibilização das informações dos PMFSs aprovados no Brasil por parte dos órgãos ambientais competentes. Essas informações são imprescindíveis para uma análise consistente em nível nacional, além de possibilitar a realização de inúmeras pesquisas sobre o manejo das florestas naturais.

\section{Conclusões}

Diversos fatores influenciam a definição de uma espécie como comercial, como o conhecimento sobre ela, a presença e abundância dessa na floresta e a demanda do mercado local e regional. As particularidades locais de cada floresta estudada são fatores que influenciam a riqueza de espécies de interesse comercial e a baixa similaridade entre as áreas de manejo.
A variação entre os nomes populares das espécies madeiras foi ampla. Essa variação é afetada pelos dialetos locais e caraterísticas das espécies, que fazem parte do conhecimento popular local. Os nomes populares aparentam ter forte relação com gêneros e famílias botânicas, podendo variar de diversas formas quanto analisados ao nível de espécie. No entanto, os nomes populares das espécies com mercado consolidado, amplamente exploradas e conhecidas, apresentaram fidelidade, sendo comuns a uma extensa região da Amazônia brasileira.

Os principais problemas de identificação estão relacionados aos gêneros e famílias de elevada riqueza de espécies e complexidade taxonômica. Por possuírem alto valor comercial e estarem presentes em diversos planos de manejo florestal sustentável da Amazônia brasileira, esses grupos devem ser tratados com atenção e ser priorizados nas pesquisas voltadas ao aprimoramento do conhecimento taxonômico.

\section{Referências}

Baraloto, C. et al. Limitations and applications of parataxonomy for community forest management in Southwestern Amazonia. Ethnobotany Research \& Applications, v. 5, p. 77-84, 2007. DOI: 10.17348/era.5.0.77-84.

Colwell. R. K. et al. Models and estimators linking individualbased and sample-based rarefaction: extrapolation and comparison of assemblages. Plant Ecology, v. 5, p. 3-21, 2011. DOI: 10.1093/ jpe/rtr044.

Ferreira, G. C. \& Hopkins, M. J. G. Manual de identificação botânica e anatômica: angelim. Belém, PA: Embrapa Amazônia Oriental, 2004. 101 p.

Janzen, D. H. Setting up tropical biodiversity for conservation through non-damaging use: participation by parataxonomists. Journal of Applied Ecology, v. 41, p. 181-187, 2004. DOI: 10.1111/j.1365-2664.2004.00879.x.

Lang, C. et al. Discrimination of taxonomic identity at species, genus and family levels using Fourier Transformed Near-Infrared Spectroscopy (FT-NIR). Forest Ecology and Management, v. 406, p. 219-227, 2017. DOI: 10.1016/j.foreco.2017.09.003.

Obermuller, F. A. et al. Guia Ilustrado e manual de arquitetura foliar para espécies madeireiras da Amazônia Ocidental. Rio Branco, AC: G. K. Noronha, 2011. 101 p.

Ribeiro, J. E. L. S. et al. Flora da Reserva Ducke: guia de identificação das plantas vasculares de uma floresta de terra-firme na Amazônia Central. Manaus, AM: INPA, 1999. 816 p.

Ter Steege, H. et al. A spatial model of tree diversity and tree density for the Amazon. Biodiversity and Conservation, v. 12, p. 22552277, 2003. DOI: 10.1023/A:1024593414624. 
The Angiosperm Phylogeny Group. An update of the Angiosperm Phylogeny Group classification for the orders and families of flowering plants: APG IV. Botanical Journal Linnieu Society, v. 181, p. 1-20, 2016. DOI: 10.1111/boj.12385.
Toledo, M. et al. Patterns and determinants of floristic variation across Lowland Forests of Bolivia. Biotropica, v. 43, n. 4, p. 405-413, 2011. DOI: $10.1111 /$ j.1744-7429.2010. 00711.x

Veríssimo, A. et al. Priority areas for establishing National Forests in the Brazilian Amazon. Conservation Ecology, v. 6, n. 1, p. 4, 2002. 